\title{
Survival of untreated advanced colorectal cancer patients
}

\author{
G.P. STATHOPOULOS
}

\author{
First Oncology Clinic, Errikos Dunant Hospital, Athens, Greece
}

Received December 20, 2010; Accepted April 14, 2011

DOI: $10.3892 / \mathrm{ol} .2011 .310$

\begin{abstract}
Colorectal cancer has specific biological characteristics that distinguish it from other malignancies. One such characteristic is its slow growth in patients in advanced stages. For the past 15 years, no effective systemic treatment has been available in clinical practice. The present study involved a retrospective evaluation of patients with advanced colorectal cancer in order to assess the median and overall survival of patients. Concurrently, the study aimed to describe the biological characteristics of this slow-growing disease and the quality of life of the patients. The key characteristic of this patient group was the lack of any systemic treatment. The study included 40 patients (25 male and 15 female, median age 67 years) who were evaluated between 1993 and 1996. Only supportive treatment was provided. One patient underwent 2 cycles of chemotherapy. Liver surgery was unsuccessfully performed on 3 patients. Two patients underwent radiofrequency once and 2 had intra-arterial treatment, also once. The results showed the median survival of patients to be 24 months (range 16-42). One-year survival was found to be $65 \%$ while the 2-year survival was found to be $25 \%$. A satisfactory quality of life was also observed. In conclusion, colorectal cancer is a slow-going malignancy, as indicated by the long-term survival of patients and the biological characteristics of the tumor.
\end{abstract}

\section{Introduction}

Colorectal cancer is a common malignancy that is surgically treated. Radiotherapy has been successfully applied in rectal cancer as adjuvant therapy and in the treatment of residual disease. Chemotherapy based on 5-fluorouracil was previously administered, but without any noteworthy effects (1-3). Chemotherapy was rendered a viable treatment modality when leucovorin was introduced into clinical practice as a 5-fluorouracil modulator $(4,5)$. Prior to this introduction, patients with advanced colorectal cancer remained untreated, since chemotherapy alone was ineffective. New drugs were only used in protocol trials. Irinotecan was regarded as a new drug that was reported to be effective in colorectal cancer

Correspondence to: Dr G.P. Stathopoulos, Semitelou 2A, 11528 Athens, Greece

E-mail: dr-gps@ath.forthnet.gr

Key words: untreated, advanced colorectal cancer when combined with leucovorin and 5-fluorouracil (6-9). During the last decade, the cisplatin analogue oxaliplatin was introduced into clinical practice, mainly for the treatment of advanced stages of the disease, and was combined with leucovorin and 5-fluorouracil or with irinotecan. The results were positive with respect to the response rate, median and overall survival. (10-16). Currently, no new cytotoxic agent has been produced for the treatment of colorectal cancer with the exception of oral capecitabine, which has been used as a substitute for leucovorin and 5-fluorouracil.

The present study involved a retrospective evaluation of 40 patients with advanced metastatic colorectal cancer in order to assess the median and overall survival of patients. Concurrently, a description was provided of the biological characteristics of this slow-growing disease as well as the quality of life of the patients as determined by their performance status (PS) .

\section{Materials and methods}

Patients. The untreated patients were randomly recruited between 1993 and 1996. Patients were untreated for the reasons that they refused to undergo cytotoxic therapy or the disease remained stable and the patients were asymptomatic. Certain patients required supportive treatment, such as antibiotics in case of infection, or blood transfusion for anemia.

Eligibility criteria. A patient histological diagnosis of colorectal cancer and advanced metastatic disease were confirmed by radiological examinations. None of the patients had undergone prior chemotherapy, although $2(5 \%)$ of the patients had received adjuvant treatment prior to the appearance of metastatic disease. Physical examination, X-rays, ultra-sound or computed tomography (CT) were performed prior to disease management. Eligibility criteria included stage IV disease, performance status of $0-2$, (World Health Organization), an expected survival of $\geq 12$ weeks and that patients be $\geq 18$ years old. Laboratory examinations were performed to evaluate disease progression. Patients were required to have adequate bone marrow reserves (leukocyte count $\geq 3500 / \mu 1$, platelet count $\geq 100,000 / \mu 1$ and hemoglobin $\geq 10 \mathrm{~g} / \mathrm{dl}$ ) and adequate renal function (serum creatinine $\leq 1.5 \mathrm{mg} / \mathrm{dl}$ and serum transaminases $\leq 3$ times the upper limit of normal). Patients exhibiting central nervous system involvement or secondary malignancy were excluded from the study. This study was conducted with the approval of the institutional review board. Written informed consent was obtained from the patients included in this study. 
Table I. Patient characteristics.

\begin{tabular}{lcr}
\hline & No. & $\%$ \\
\hline Patients evaluated & 40 & 100.0 \\
Median age (years) & 67 & \\
(range) & $(32-87)$ & \\
Gender & & 62.5 \\
Male & 25 & 37.5 \\
Female & 15 & \\
Disease stage & & 100.0 \\
IV & 40 & \\
PS & & 82.5 \\
0-1 & 33 & 17.5 \\
2 & 7 & \\
Histology adenocarcinoma & & 100.0 \\
Moderate differentiation & 40 & \\
Site of metastasis & & 20.5 \\
Liver & 18 & 25.0 \\
Lung & 3 & \\
Abdomen & 10 & \\
Bone & 1 & \\
Multiple & 8 & \\
\hline
\end{tabular}

Table II. Treatment.

\begin{tabular}{lcc}
\hline Treatment & No. & $\%$ \\
\hline Chemotherapy (2 cycles) & 1 & 2.5 \\
Liver surgery & 3 & 7.5 \\
Radiotherapy, skeleton & 1 & 2.5 \\
Radiofrequency & 2 & 5.0 \\
Intra-arterial & 2 & 5.0 \\
Chemo-embolization & 2 & 5.0 \\
Total & 11 & 27.5 \\
\hline
\end{tabular}

Following the baseline tests, patients were followed up via a clinico-laboratory evaluation that included a physical examination, ECG, full blood count, liver and renal function tests, and urine analysis. Radiological tests were performed where disease progression was indicated by clinical signs, otherwise the tests were performed every six months.

\section{Results}

Patients. A total of 40 patients with histologically confirmed adenocarcinomas of moderate differentiation were included and evaluated. Table I shows the patient characteristics at baseline.

Only two patients were administered with adjuvant chemotherapy (leucovorin and 5-fluorouracil). This treatment had been administered between 1993 and 1996 when adjuvant chemotherapy with irinotecan or oxaliplatin was not yet available. All 40 patients had metastatic disease when the evaluation
Table III. Survival in months.

Median survival

$24(95 \%$ CI, 16-42)

\begin{tabular}{lc}
\hline Range & $5-42$ \\
One-year & 26 patients $(65.0 \%)$ \\
2-year & 10 patients $(25.0 \%)$ \\
Alive at the end of the study & 21 patients $(52.5 \%)$
\end{tabular}

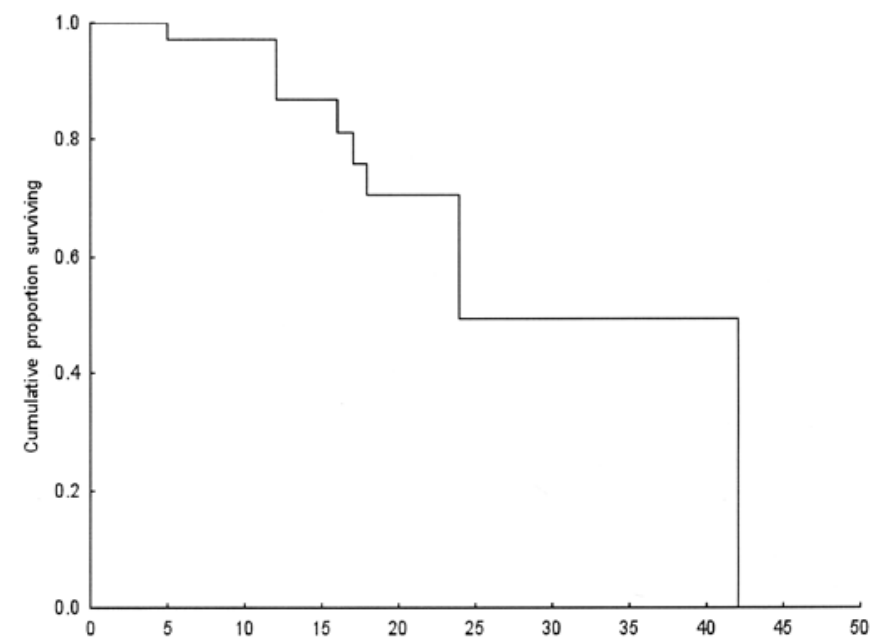

Figure 1. Kaplan-Meier survival curve in months.

and follow-up were commenced. Systemic treatment was not administered to the majority of patients (29 patients, $72.5 \%$ ). Of the remaining 11,1 patient underwent 2 courses of chemotherapy, and 2 patients underwent liver surgery due to liver metastases. The 2 patients undergoing liver resection showed residual disease within 3 months after surgery and 1 patient showed liver recurrence, also within 3 months. Intra-arterial chemotherapy was administered once to 2 patients and chemo-embolization was administered to 2 further patients, also once. Radiotherapy was administered to 2 patients with temporary liver deposit reduction (Table II).

Survival. The median survival for the 40 patients was 24 months (range 5-42). A total of 26 patients (65\%) survived for one year. After 2 years, 10 patients had survived (25\%; Table III). The quality of life of these 10 patients was satisfactory based on the PS, which remained at 0-1.

Statistical analysis. The Kaplan-Meier method was used for survival distribution estimation (Fig. 1).

\section{Discussion}

Colorectal cancer is a slow-growing malignancy. In the majority of patients, the result of chemotherapy in advanced metastatic disease is stabilization (17). According to the RECIST criteria, stable disease is defined as a tumor reduction of less than $30 \%$ and tumor progression of less than $20 \%$ (18). Where evaluation by CT after treatment showed stable 
disease, the result may be attributed to either: i) the effect of chemotherapy, which inhibited disease progression, or ii) the biological characteristics of the tumor, which define how rapidly it develops. It is difficult to verify which interpretation applies in routine clinical practice. Relevant data are required to determine whether chemotherapy plays a significant role in regulating disease progression. One example of how data may be gathered involves a comparison of treated versus non-treated patients in a randomized trial. A study by Cunningham et al, which compared chemotherapy with irinotecan plus supportive care versus supportive care alone, showed that overall survival was significantly higher in the irinotecan group $(\mathrm{p}=0.001)(18)$. Results by these authors showed that one-year survival for the irinotecan group was $36.2 \%$ compared with $13.8 \%$ for the supportive care group. These percentages of one-year survival for each group are lower than those obtained in our study. The reason for this discrepancy is that the previous study included the time period after the administration of second-line treatment (18). On the other hand, the results of the study by Cunningham et al (18) indicate the advantage of chemotherapy in colorectal cancer. The aim of our study was to contribute to a better understanding of the biology of colorectal cancer. Our findings indicate that this is a slow-growing disease with a $65 \%$ survival rate after 1 year, and a $25 \%$ survival rate after 2 years (10/40 patients). Local treatment administered to $11 / 40$ patients $(27.5 \%)$ contributed little to overall survival.

The long-term survival of patients with metastatic advanced colorectal cancer without systemic treatment may be due to the biological characteristics of this tumor, indicating that it is a slow-growing malignancy. These findings are useful in obviating long-term or unstoppable chemotherapy when patients are in stable disease.

\section{References}

1. Gastrointestinal Tumor Study Group: Adjuvant therapy for colon cancer: results of a prospectively randomized trial, $\mathrm{N}$ Engl J Med 310: 737-740, 1984.

2. Mulcahy HE, Toner M, Patchett SE, et al: Identifying stage B colorectal cancer patients at high risk of tumor recurrence and death. Dis Colon Rectum 40: 326-336, 1997.

3. Moertel CG: Chemotherapy of gastrointestinal cancer In: Recent Advances in Cancer Treatment. Tajnon HJ and Staquet MJ (eds.) New York, Raven Press, pp 311-323, 1997.

4. Wolmark N, Rockette H, Fisher B, et al: The benefit of leucovorin-modulated fluorouracil as postoperative adjuvant therapy for primary colon cancer: results from National Surgical Adjuvant Breast and Bowel Project. Protocol C-03. J Clin Oncol 11: 1879-87, 1993
5. Francini GP and Lorenzini L: Folinic acid and 5-fluorouracil as adjuvant chemotherapy in colon cancer. Gastroenterology 106: 899-906, 1994.

6. De Gramont A, Vignoud J, Tournigand C, et al: Oxaliplatin with high-dose leucovorin and 5-fluorouracil 48-hour continuous infusion in pretreated metastatic colorectal cancer.Eur JCancer 33: 214-219, 1997.

7. Rougier P, Van Cutsem E, Bajetta E, et al: Randomised trial of irinotecan versus fluorouracil by continuous infusion after fluorouracil failure in patients with metastatic colorectal cancer. Lancet 352: 1407-1412, 1998.

8. Sargent DJ, Niedzwiecki D, O' Conell MJ, et al: Recommendation for caution with irinotecan, fluorouracil, and leucovorin for colorectal cancer. N Engl J Med 345: 144-145, 2001.

9. De Gramont A, Figer A, Seymour M, et al: Leucovorin and fluorouracil with or without oxaliplatin as first-line treatment in advanced colorectal cancer. J Clin Oncol 18: 2938-2947, 2000.

10. Scheithauer W, Kornek GV, Raderer M, et al: Combined irinotecan and oxaliplatin plus granulocyte colony-stimulating factor in patients with advanced fluoropyrimidine/leucovorinpretreated colorectal cancer. J Clin Oncol 17: 902-906, 1999.

11. Rothenberg ML: Efficiacy of oxaliplatin in the treatment of colorectal cancer. Oncology 14: 9-14, 2000.

12. Souglakos J, Mavroudis D, Kakolyris S, et al: Triplet combination with irinotecan plus oxaliplatin plus continuous-infusion fluorouracil and leucovorin as first-line treatment in metastatic colorectal cancer: a multicenter phase II trial. J Clin Oncol 20: 2651-2657, 2002.

13. Scheithauer W, Kornek GV, Raderer M, et al: Randomized multicenter Phase II trial of two different schedules of capecitabine plus oxaliplatin as first-line treatment in advanced colorectal cancer. J Clin Oncol 21: 1307-1312, 2003.

14. Goldberg RM, Sargent DJ, Morton RF, et al: A randomized controlled trial of fluorouracil plus leucovorin, irinotecan and oxaliplatin combinations in patients with previously untreated metastatic colorectal cancer. J Clin Oncol 22: 23-30, 2004.

15. Stathopoulos GP, Rigatos SK, Stathopoulos JG, Xynotroulas JP and Dimou E: Efficacy and tolerability of oxaliplatin plus irinotecan 5-fluorouracil and leucovorin regimen in advanced stage colorectal cancer patients pretreated with irinotecan, 5-fluorouracil and leucovorin. AJ Clin Oncol 28: 565-569, 2005.

16. Stathopoulos GP, Rigatos SK, Malamos N, et al: Long term survival of patients with advanced colorectal cancer may not be due to the response to chemotherapy. Oncology Rep 12: 1295-1300, 2004.

17. Therasse P, Arbuck SG, Eisenhower E, et al: New guidelines to evaluate the response to tumor in solid tumors. JNCI 92: 205-216, 2000

18. Cunningham D, Pyrhonen S, James RD, et al: Randomised trial of irinotecan plus supportive care versus supportive care alone after fluorouracil failure for patients with metastatic colorectal cancer. Lancet 31 (353): 1275-1276, 1999. 